\title{
Phronesis
}

\section{De la cohérence épistémologique de la posture collaborative}

\section{Joëlle Morissette, Maria Pagoni et Matthias Pépin}

Volume 6, numéro 1-2, 2017

Les recherches collaboratives en éducation et en formation : référents théoriques, outils méthodologiques et impacts sur les pratiques professionnelles

URI : https://id.erudit.org/iderudit/1040213ar

DOI : https://doi.org/10.7202/1040213ar

Aller au sommaire du numéro

Éditeur(s)

Université de Sherbrooke

Champ social éditions

ISSN

1925-4873 (numérique)

Découvrir la revue

Citer ce document

Morissette, J., Pagoni, M. \& Pépin, M. (2017). De la cohérence épistémologique de la posture collaborative. Phronesis, 6(1-2), 1-7.

https://doi.org/10.7202/1040213ar d'utilisation que vous pouvez consulter en ligne. 


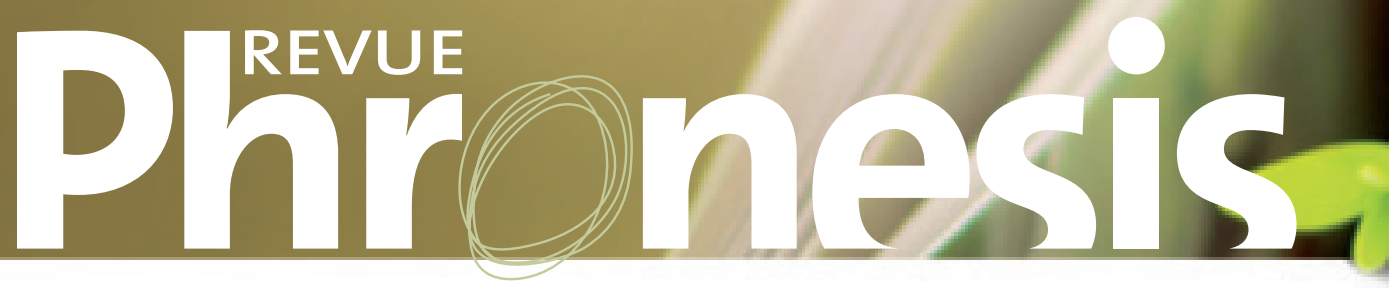

Volume 6, numéro 1-2 | 2017

\section{LES RECHERCHES COLLABORATIVES EN ÉDUCATION ET EN FORMATION}

Numéro coordonné par :

\section{Joëlle Morissette,}

Professeure,

Université de Montréal,

\section{Maria Pagoni,}

Professeure,

Université de Lille

Matthias Pépin,

Chercheur postdoctoral

Université du Québec à Trois-Rivières

RÉFÉRENTS THÉORIQUES, OUTILS MÉTHODOLOGIQUES ET IMPACTS SUR LES PRATIQUES PROFESSIONNELLES QUELLE PLACE POUR LES FONDEMENTS DE L'ÉDUCATION DANS LE TRAVAIL ÉDUCATIF AUJOURD'HUI ? 


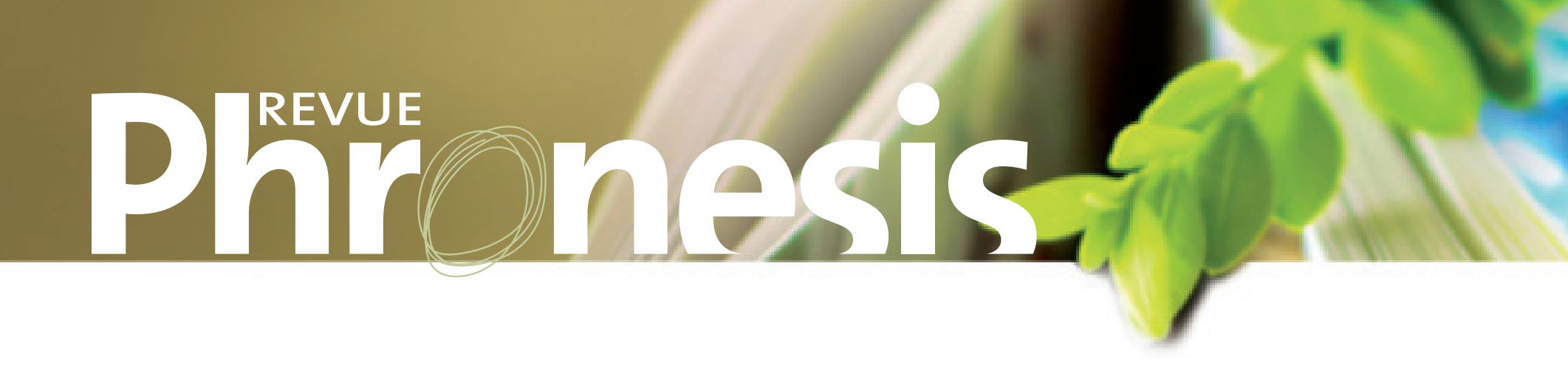

\section{De la cohérence épistémologique de la posture collaborative}

Depuis le début des années 1980, un courant important propose d'établir une dialectique plus équilibrée entre les préoccupations du monde de la recherche et celles du monde de la pratique professionnelle. C'est dans ce contexte que des auteurs tels que Lieberman (1986) ont proposé de faire de la recherche avec les professionnels, plutôt que seulement sur eux, invitant à définir des manières de faire la recherche qui leur seraient aussi utiles. Fortes d'une nouvelle légitimité, des recherches dites collaboratives commencent alors à se développer en poursuivant une double finalité, concourant à asseoir leur pertinence à la fois pour les milieux de recherche, à travers la production de savoirs scientifiques, et pour les milieux de pratique, par la formation des professionnels. En conséquence, les dispositifs de recherche collaborative se sont multipliés, particulièrement dans les facultés universitaires qui ont une vocation de formation professionnelle (Anadón, 2007).

En Amérique du Nord, deux grandes tendances se démarquent en langue française, selon les visées poursuivies par les recherches collaboratives (Morrissette, 2013) : parfois qualifiées de recherches-action collaboratives, certaines adoptent une stratégie d'intervention visant le changement à travers une démarche de résolution de problèmes susceptible de contribuer à améliorer une situation jugée problématique par un groupe de professionnels (Bourassa, Leclerc \& Fournier, 2010; Savoie-Zajc \& Lanaris, 2005); d'autres visent plutôt l'exploration et l'explicitation d'un aspect de la pratique à partir de la compréhension en contexte des professionnels, dans une démarche de co-construction d'un savoir professionnel qui soit le produit combiné et inédit des logiques et enjeux de la communauté des chercheurs et de celle des praticiens (Desgagné, 1998; Diédhiou, 2013). Les recherches collaboratives qui se sont développées dans le monde francophone européen se trouvent pour leur part en lien avec les travaux sur l'observation des pratiques effectives des professionnels de l'éducation et leur analyse conjointe (Altet, Bru, Blanchard-Laville, 2012; Blanchard-Laville \& Fablet 1998; Perrenoud 1996). Des recherches se sont aussi élaborées en lien avec les modèles théoriques de l'analyse de l'activité professionnelle et en particulier ceux de la didactique professionnelle (Pastré 2011; Pastré, et al., 2006; Vinatier 2009) et de la clinique de l'activité (Clot, 1999; Roger, 2007).

En ce qui concerne le champ de l'éducation et de la formation en particulier, les recherches collaboratives se sont inspirées des questionnements concernant le processus d'apprentissage "sur le tas», soit les manières qu'ont les professionnels de résoudre des problèmes de pratique en contexte, dans le feu de l'action, et qui concourent à l'élaboration de leur répertoire de savoirs professionnels (Dewey 2010/1934; Schön, 1983). Jusqu'ici, les recherches collaboratives ont suscité des questionnements concernant les conditions et les effets de leur mise en place (Kahn, Hersant \& Orange-Ravachol, 2010; Yvon \& Durand, 2011). Différents référents ont été mobilisés, mettant de l'avant des outils méthodologiques contribuant à la co-construction de savoirs dans la double logique de la recherche et du développement professionnel, à travers des activités réflexives (Desgagné, 2007; Morrissette, 2012; Vinatier, 2007) ou des entretiens de type auto-confrontation simple ou croisée conduits avec I'aide d'enseignants-chercheurs (Clot et al., 2001; Pagoni, 2014). Des réflexions ont été conduites autour du rôle régulateur du contrat collaboratif en cours de démarche (Bednarz et al., 2012), d'une action conjointe conduite en contexte (Filliettaz, 2010), d'une «ingénierie coopérative» où chercheurs et professionnels agissent ensemble dans une posture commune de production didactique (Sensevy et al., 2013) ou, encore, d'une «enquête collaborative» (Imbert \& Durand, 2010). Les recherches ont également interrogé le caractère négocié des savoirs produits entre les deux communautés impliquées (Desgagné \& Bednarz, 2005; Ducharme et al., 2007; Morrissette \& Desgagné, 2009; Morrissette et al., 2012), ainsi que les enjeux épistémologiques soulevés par l'articulation entre savoirs d'expérience et savoirs scientifiques (Kahn, 2010; Perrenoud et al., 2008). 
Ce numéro thématique de la revue Phronesis fait suite à un symposium intitulé "Observation et mise en mots des pratiques dans le cadre des recherches collaboratives: Quelles finalités? Quels dispositifs? Quels impacts?» (Structure Fédérative OPÉEN \& ReForm, Université de Nantes, 8-10 juin 2016). II rassemble des réflexions épistémologiques et méthodologiques sur les recherches collaboratives qui se sont développées en Europe et en Amérique du Nord pour interroger la collaboration entre chercheurs et professionnels dans le cadre des recherches en éducation et en formation. Les textes qui composent ce numéro, dont la provenance s'étend bien au-delà des participants au symposium qui en constitue l'origine, visent ainsi à questionner les finalités, les dispositifs et les impacts de recherches collaboratives qui se centrent sur les pratiques d'éducation et de formation dans différents champs professionnels. Par-là, ce dossier entend ainsi contribuer au domaine des recherches collaboratives en réfléchissant aux difficultés qu'elles posent selon les étapes des démarches qu'elles empruntent, de même qu'aux enjeux qu'elles suscitent (éthiques, pragmatiques, théoriques, etc.), et ce, afin d'alimenter un débat constructif alors qu'elles connaissent une popularité grandissante. Il entend également contribuer à la mise en place d'un dialogue fécond entre les travaux de recherche en Europe et en Amérique du nord dans ce domaine ainsi qu'à l'analyse de leurs influences réciproques.

Avant de présenter plus en détail ces textes s'imposent quelques observations transversales sur ceux-ci. Le premier constat qui saute rapidement aux yeux renvoie à la nature hétéroclite, sinon éclatée, du concept même de recherche collaborative et des contributions qui s'en revendiquent. Chaque auteur ou collectif d'auteurs présente de ce fait sa propre compréhension de la recherche collaborative, empruntant tantôt à la recherche-action, à la recherche-intervention, à la recherche participative et aux lesson studies. Si des approches structurées de la recherche collaborative ont bien été proposées, notamment celle de Desgagné $(1997,1998)$ il y a vingt ans déjà, force est de constater que les référents théoriques, tout comme les outils méthodologiques des recherches collaboratives contemporaines se sont multipliés. Les textes qui composent ce numéro ancrent en effet leurs réflexions à des traditions aussi variées que la didactique professionnelle, la psychanalyse ou encore la sociologie du travail pour n'en citer que quelques-unes. Là encore, à travers ces contributions, la recherche collaborative n'apparaît pas comme une méthodologie de collecte de données uniforme. En effet, les auteurs s'appuient sur diverses méthodes et outils, tels les entretiens semi-directifs ou d'auto-confrontation qui servent aussi d'activités réflexives, en fonction des enjeux de leurs terrains et de leurs objets de recherche. Loin de présenter un corpus théorique ou méthodologique homogène, ce numéro sert en définitive de prisme mettant au jour un spectre de la recherche collaborative plus diffracté que ce qui était attendu.

Au-delà la diversité dont nous venons de faire état, se profile pourtant une ligne directrice guidant toutes les contributions de ce numéro, et c'est là le second constat qui ressort d'une lecture transversale des textes. L'unité de ce qu'il conviendrait d'appeler les recherches collaboratives se dessine ainsi au plan épistémologique, comme une posture, une manière de concevoir la recherche de terrain. Tous les auteurs, en dépit des particularités qui caractérisent leur opérationnalisation de l'idée de "faire de la recherche collaborative», s'entendent ainsi sur au moins trois points centraux. Le premier tient au fait que tous adhèrent à l'idée d'impliquer les acteurs dans leurs recherches, comme l'appelait de ses vœux Lieberman (1986). Cette volonté de travailler avec les acteurs de la recherche n'est pas, alors, sans amener son lot de questionnements méthodologiques, qui rejoignent à certains égards ceux de la recherche-action (Hess, 1981; Dolbec \& Clément, 2004) et de l'ethnographie collaborative (Lassiter, 2005), deux autres traditions de recherche qui réservent une place de choix aux participants dans la conduite de la recherche. Ceci dit, dans les recherches collaboratives, il ne s'agit pas tant de faire jouer aux participants les rôles normalement dévolus au chercheur à toutes les étapes de la recherche, comme c'est le cas d'une certaine conception de la recherche-action, à travers des spirales de résolution de problèmes tirant leur origine d'une problématique plus générale vécue par les participants et pour laquelle ils font appel à un chercheur. II ne s'agit pas non plus, comme avec l'ethnographie collaborative, d'entamer un rapport dialogique avec les participants au fur et à mesure que s'écrit le "texte du savoir » qui fait suite aux observations de terrain, dans l'optique de produire un texte qui soit pertinent à la fois pour la recherche et pour les participants (Lassiter, 2005).

Quelle est alors la particularité de la posture collaborative qui unit les contributions de ce numéro? On peut trouver un élément de réponse dans le fait que les recherches collaboratives n'adoptent pas délibérément une visée interventionniste, mais bien plutôt une visée compréhensive. Ceci constitue un élément tout à fait fondamental, surtout si l'on considère que les recherches collaboratives dont témoignent les articles de ce numéro sont conduites dans des milieux de pratique, par définition orientés vers l'action et la recherche de solutions concrètes à leurs problèmes. On peut en effet penser que si des acteurs acceptent de collaborer avec un chercheur (ou une équipe de chercheurs), c'est parce qu'ils veulent des réponses à leurs questions. Or, le chercheur, de son côté, veut avant tout comprendre les manières de faire des professionnels qu'il a choisi d'étudier, manières de faire façonnées par les contraintes, exigences et possibilités des contextes et du système de pratiques dans lequel elles s'inscrivent. Le chercheur se trouve alors en position délicate. S'il veut éviter de tomber dans le piège de celui qui sait, qui a toutes les réponses aux questions que se posent les praticiens, ce qui l'empêcherait de toute façon d'accéder à leur savoir qu'il est précisément venu chercher (Shulman, 1986), il doit se faire plus stratégique, tout en cherchant à combler les attentes légitimes des participants qui lui donnent de leur temps, qui lui donnent un accès privilégié à leur compréhension en contexte 
de l'objet de préoccupation mutuelle. Ceci étant, brandir l'argument d'un engagement au service de l'avancement de la connaissance ne saurait suffire à lui seul à convaincre des professionnels, déjà bien occupés, à s'hasarder dans un processus de recherche collaborative, demandant parfois un investissement important.

Comment s'en sortir, dès lors? C'est sans doute là qu'intervient le second point central qui rejoint tous les textes de ce numéro. En conséquence directe avec l'idée de faire de la recherche avec les participants, tous les auteurs s'entendent en effet sur la double finalité de la recherche collaborative : elle se doit d'être pertinente à la fois pour le monde de la recherche et pour le monde de la pratique; la posture collaborative ayant vocation de pont entre deux mondes historiquement, et à certains égards étrangement, séparés. À la fonction de production de connaissances scientifiques propre à toute recherche s'ajoute ainsi une volonté de développement professionnel des acteurs engagés dans la collaboration. Si les moyens diffèrent d'une recherche à l'autre, la sollicitation de la réflexivité des participants ressort invariablement comme la voie de développement professionnel privilégiée, dans la lignée du praticien réflexif initiée par Schön (1983). II s'agit donc, pour le chercheur, d'imaginer un dispositif qui lui permette de stimuler la réflexivité des professionnels avec lesquels il a choisi de collaborer. C'est ce dispositif réflexif - des groupes de réflexion, de co-développement professionnel (Payette et Champagne, 2010), des entretiens d'autoconfrontation, etc. - qui permettra d'assurer à la fois le développement professionnel des acteurs et la production de nouvelles connaissances, lesquelles s'appuient précisément sur la réflexivité des acteurs. Au départ de la recherche collaborative, c'est donc la promesse d'activités réflexives au service de la formation continue qui permet d'obtenir la collaboration des acteurs.

Si l'on fait la synthèse de deux points de convergence des textes qui constituent ce numéro, on peut dégager à nouveau deux constats importants. D'une part, adopter une visée compréhensive, comme caractéristique de la posture collaborative, ne sous-entend pas que le chercheur ne puisse pas viser aussi une transformation des pratiques des acteurs. D'ailleurs, c'est bien là un effet souhaité et attendu du dispositif réflexif mis en place aux fins d'un développement professionnel des acteurs engagés dans la collaboration. La subtilité tient au fait que ce n'est pas le changement ou encore le développement professionnel en lui-même qui fait l'objet de l'intérêt du chercheur; il s'agit en effet plutôt d'un résultat de la sollicitation de la réflexivité des acteurs qui, elle, constitue le support à la production de nouvelles connaissances scientifiques. D'autre part, adopter une visée compréhensive tout en orientant le dispositif réflexif vers un développement professionnel implique de s'entendre avec les acteurs sur ce qui fera l'objet à la fois d'une attention de recherche et d'une attention de développement professionnel, de même que la contribution attendue de chacun dans ce processus. Autrement dit, et c'est là le troisième point qui rejoint tous les textes et qui nous servira à proposer un angle particulier pour les présenter, qui dit collaboration de recherche dit négociation d'un contrat collaboratif (Bednarz et al., 2012) entre le chercheur et les participants qui s'engagent dans la collaboration de recherche. Tous les textes, chacun à leur manière, interrogent la construction, la négociation et le maintien d'une entente plus ou moins formelle qui régisse les interactions et l'engagement de chacun dans la collaboration.

\section{À propos des textes}

Les textes composant ce numéro de Phronesis sont regroupés en quatre grandes catégories qui suivent la chronologie d'une recherche collaborative, depuis sa planification jusqu'à la production des savoirs visés.

\section{Les conditions des recherches collaboratives}

Quatre textes interrogent les conditions des recherches collaboratives, le choix réciproque des acteurs ainsi que les enjeux éthiques, politiques, scientifiques et relationnels qui se posent dans le cadre de cette collaboration. Pierre Lapointe et Joëlle Morrissette proposent à ce propos une analyse du travail de médiation qui se met en place entre chercheurs et professionnels pendant la phase de définition du problème, à partir de deux exemples de projets de recherche menés au Québec. Corinne Marlot, Marie Toullec-Théry et Marc Daguson mettent en exergue les conditions du rapprochement entre chercheurs et professionnels ainsi que les modalités de négociation de l'objet de recherche / de formation qui en découle en faisant appel au concept d'acculturation réciproque. Daniel Martin et d'Anne Clerc-Georgy questionnent, quant à eux, la variabilité des postures des chercheurs à partir de l'analyse de plusieurs projets de lesson study concernant l'enseignement de mathématiques et de physique aussi bien dans le primaire que dans le secondaire. Enfin, en adoptant un point de vue psychanalytique, Arnaud Dubois et Antoine Kattar s'intéressent à la place accordée aux acteurs directement concernés par l'objet de la recherche (public d'adolescents qui ont subi l'exclusion dans trois collèges différents) et posent la question de la dynamique du transfert et du contre-transfert dans la relation au thème de la recherche et dans la relation avec les sujets engagés. 
Quatre textes, s'intéressent à la dynamique d'accompagnement qui se met en place dans le cadre d'une recherche collaborative, en insistant plus particulièrement sur le processus réflexif engendré et les méthodologies utilisées à cet effet. Bruno Bourassa, France Picard, Yann Le Bossé et Geneviève Fournier interrogent le rôle central de la posture d'accompagnement dans la recherche collaborative en s'appuyant sur une expérience de 25 ans dans ce type de recherches auprès des professionnels de l'intervention socio-éducative et en montrant le processus réflexif qui se met en place, source d'apprentissage et de développement professionnel, avec l'aide du chercheur, à partir d'un incident critique. Nina Asloum et Guy Asloum présentent la place des recherches collaboratives dans la conduite et l'accompagnement du changement à partir d'une méta-analyse d'un ensemble de textes publiés dans un ouvrage collectif coordonné par une unité mixte de recherche qui travaille sur cette thématique. La recherche collaborative présentée par Vincent Enrico, portant sur l'activité de la société ADL (Aide à Domicile en Limousin), montre comment la mise à jour de l'organisation du travail quotidien engageant différentes cultures professionnelles (celle du technicien, du corps médical et du travailleur social) conduit à une réflexivité dans le sens d'une capacité de chacun à réfléchir à son rapport à l'écrit restitué et aux autres professionnels. Enfin, Valérie Melin convoque l'approche de la recherche biographique pour analyser les effets d'un atelier biographique destiné à des élèves, anciens décrocheurs, dans le changement de posture des enseignants et des élèves qui y participent, par la production des savoirs issus de cet atelier.

\section{La place des points d'achoppement dans la construction des objets de questionnement}

Deux textes se centrent plus spécifiquement sur la place des points d'achoppement (controverses, conciliation des points de vue) dans le processus de co-construction des objets de questionnement par les chercheurs et les professionnels. Ainsi, Maria Pagoni et Catherine Boyer s'appuient sur l'analyse d'un entretien d'auto-confrontation collectif avec des enseignants expérimentant un dispositif innovant en pédagogie Freinet, pour interroger la place du questionnement des chercheurs et des débats installés dans l'émergence de l'objet de recherche. Matthias Pepin et Serge Desgagné convoque, quant à lui, le concept de double vraisemblance pour décrire la négociation entre chercheur et professionnel au vu de l'élaboration des pratiques pédagogiques accompagnant la mise en place d'un magasin scolaire, par les élèves, visant l'apprentissage à s'entreprendre.

\section{Les bénéfices mutuels des recherches pour les deux communautés}

Les quatre derniers textes s'intéressent aux bénéfices mutuels de ces recherches pour les deux communautés, aussi bien en termes de développement professionnel qu'en termes de construction des connaissances. Isabelle Nizet et Réjane Monod Ansaldi montrent comment trois concepts intermédiaires, celui de valuation des savoirs, de brokering et d'objet frontière, sont utilisés pour caractériser les savoirs produits par deux recherches collaboratives sur la formation des enseignants et construire leur légitimité pour les deux communautés impliquées. Christine Pierrisnard explique comment une recherche collaborative portant sur la discussion à visée démocratique et philosophique contribue, par les entretiens d'auto-explicitation, au développement de l'expertise aussi bien des praticiens que du chercheur et à la théorisation de leurs connaissances. À travers une démarche socio-clinique, Corinne Rougerie questionne quant à elle l'accueil du chercheur dans un centre social français et le processus réflexif qui s'installe pour tous les acteurs impliqués, moteur de développement professionnel réciproque. Enfin, en situant la recherche collaborative dans une perspective écologique visant à rapprocher deux systèmes de savoirs et de pratiques (des scientifiques et des professionnels), Geneviève Lessard, Julie Bergeron, Stéphanie Demers et Nathalie Anwandter présentent combien cette approche de la recherche devient source de développement professionnel et d'émancipation aussi bien pour les praticiens que pour les chercheurs, en interrogeant ainsi les contraintes qui régissent le travail dans les deux communautés.

\section{Références bibliographiques}

Altet, M., Bru, M. \& Blanchard-Laville, C. (2012). Observer les pratiques enseignantes. Paris : L'Harmattan.

Anadón, M. (dir.) (2007). La recherche participative : multiples regards. Québec: Presses de l'Université du Québec.

Bednarz, N., Desgagné, S. Maheux, J.-F. \& Savoie-Zajc, L (2012). La mise au jour d'un contrat réflexif comme régulateur de démarches de recherche participative : le cas d'une recherche-action et d'une recherche collaborative. Recherches en éducation, 14, 129-152. Téléaccessible à : http://www.recherches-en-education.net/IMG/pdf/REE-no14.pdf

Blanchard-Laville, C.\& Fablet D. (1998). Analyser les pratiques professionnelles. Paris : L'Harmattan. 
Bourassa, B., Leclerc, C. \& Fournier, G. (2010). Une recherche collaborative en contexte d'entreprise d'insertion : de l'idéal au possible. Recherches qualitatives, 29(1), 140-164. Téléaccessible à : http://www.recherche-qualitative.qc.ca/documents/ files/revue/edition reguliere/numero29\%281\%29/RO Bourassa al.pdf

Clot, Y. (1999). La fonction psychologique du travail. Paris : PUF.

Clot, Y., Faïta, D., Fernandez, G. \& Scheller, L. (2001). Entretiens en autoconfrontation croisée : une méthode en clinique de I'activité. Education Permanente, 146(1), 17-25.

Desgagné, S. (2007). Le défi de coproduction de savoir en recherche collaborative : autour d'une démarche de reconstruction et d'analyse de récits de pratique enseignante. Dans M. Anadon (dir.) La recherche participative : multiples regards (p. 89-121). Québec: Presses de l'Université du Québec.

Desgagné, S. (1998). La position du chercheur en recherche collaborative : illustration d'une démarche de médiation entre culture universitaire et culture scolaire. Recherches qualitatives, 18, 77-105.

Desgagné, S. (1997). Le concept de recherche collaborative : I'idée d'un rapprochement entre chercheurs et praticiens enseignants. Revue des sciences de l'éducation, 23(2), 371-393.

Desgagné, S. \& Bednarz, N. (2005). Médiation entre recherche et pratique en éducation : faire de la recherche «avec» et plutôt que «sur» les praticiens. Revue des sciences de liéducation, 31 (2), 245-258.

Dewey J. (1934/2010). L'art comme expérience. Paris : Gallimard.

Diédhiou, S.B.M. (2013). Façons de faire l'évaluation formative d'enseignants de français sénégalais : une analyse de leurs savoirs pratiques en contexte d'effectifs pléthoriques au Lycée. Sarrebruck, CH : Les Éditions universitaires européennes.

Dolbec, A., \& Clément, J. (2004). La recherche-action. Dans T. Karsenti \& L. Savoie-Zajc (dir.). La recherche en éducation : étapes et approches (p. 181-208). Sherbrooke : Éditions du CRP.

Ducharme, D., Leblanc, R., Bourassa, M. et Chevalier, J. (2007). La recherche collaborative en milieu scolaire : un travail diacculturation. Éducation et francophonie, 35(2), 217-232. Téléaccessible à : http://www.acelf.ca/c/revue/pdf/ $\underline{X X X V 2217 . p d f}$

Filliettaz, L. (2010). Les formes situées de l'explicitation de l'action comme dévoilement des savoirs issus de la pratique : le cas des interactions en formation professionnelle initiale. Recherches en éducation, numéro spécial 1, "Savoirs et collaborations entre enseignants et chercheurs en éducation », 97-110. Téléaccessible à : http://www.recherches-en-education. net/IMG/pdf/REE-HS-no1.pdf

Hess, R. (1981). Lewin et la recherche-action. Dans La sociologie d'intervention (p. 99-109). Paris: Presses Universitaires de France.

Imbert, P. \& Durand, M. (2010). Utilitarisme et anti-utilitarisme dans le travail : pour une éthique en acte dans l'enquête collaborative sur le travail éducatif et la formation professionnelle. Actes du Congrès international de l'AREF, 13-16 septembre 2010, Université de Genève. Téléaccessible à : https://plone.unige.ch/aref2010/symposiums-longs/coordinateurs-en-r/ dimensions-cachees-dimensions-clandestines-du-travail-des-concepts-pour-penser-le-travail-educatif/Utilitarisme\%20 et\%20anti-utilitarisme.pdf/view

Kahn, S. (2010). Les réformes contre le redoublement ou les limites des recherches non collaboratives. Dans S. Kahn, M. Hersant \& D. Orange Ravachol (dir.), Recherches en éducation, hors série, 1, 71-84. Téléaccessible à : http://www.recherchesen-education.net/IMG/pdf/REE-HS-no1.pdf

Kahn, S., Hersant, M. \& Orange Ravachol, D. (2010). Savoirs et collaborations entre enseignants et chercheurs en éducation. Recherches en éducation, hors série, 1, 147 p. Téléaccessible à : http://www.recherches-en-education.net/IMG/pdf/REEHS-no1.pdf

Lassiter, L. E. (2005). The Chicago guide to collaborative ethnography. Chicago, Ill.: University of Chicago Press.

Lieberman, A. (1986). Collaborative research: Working with, not working on... Educational Leadership, 43(5), $29-32$.

Morrissette, J. (2013). Recherche-action et recherche collaborative : quel rapport aux savoirs et à la production de savoirs? Nouvelles pratiques sociales, 25(2), 35-49. Téléaccessible à : http://www.erudit.org/revue/nps/2013/v25/n2/index.html

Morrissette, J. (2012). Faire cas de sa pratique enseignante dans le cadre d'une approche collaborative. Travail et apprentissage, 9, 200-214. 
Morrissette, J., Mottier Lopez, L. \& Tessaro, W. (2012). La production de savoirs négociés dans deux recherches collaboratives sur l'évaluation formative. Dans L. Mottier Lopez \& G. Figari (dir.), Modélisations de l'évaluation en éducation (p. 27-43). Bruxelles : De Boeck, coll. Raisons éducatives.

Morrissette, J. \& Desgagné, S. (2009). Le jeu des positions de savoir en recherche collaborative : une analyse. Recherches qualitatives, 28 (2), p. 118-144. Téléaccessible à : http://www.recherche-qualitative.qc.ca/documents/files/revue/edition reguliere/numero28\%282\%29/morrissette\%2828\%292.pdf

Pagoni, M. (2014). Construire la signification des situations en conseil de coopérative. Quel questionnement de l'enseignant? In M. Pagoni (dir.), Ecole(s) et culture(s). Savoirs scolaires, pratiques sociales et significations (pp. 259-278). Bruxelles : Peter Lang, coll. Transversales.

Pastré, P. (2011). La didactique professionnelle. Approche anthropologique du développement chez les adultes. Paris : PUF.

Pastré, P., Mayen P. \& Vergnaud, G. (2006). La didactique professionnelle. Revue Française de Pédagogie, 154, 145-198.

Payette, A. \& Champagne, C. (2010). Le groupe de codéveloppement professionnel. Québec: Presses de I'Université du Québec.

Perrenoud, P., Altet, M., Lessard, C. \& Paquay, L. (2008). Conflits de savoirs en formation des enseignants : entre savoirs issus de la recherche et savoirs issus de l'expérience. Bruxelles: De Boeck, coll. Perspectives en éducation et formation.

Roger, J.-L. (2007). Refaire son métier. Essai de clinique de l'activité. Ramonville Saint-Agne : Erès.

Savoie-Zajc, L. \& Lanaris, C. (2005). Regards et réflexions d'une communauté face au problème de l'abandon scolaire. Le cas d'une recherche dans une école secondaire de l'Outaouais. Revue des sciences de l'éducation, 31(2), 297-316. [Téléaccessible à : https://www.erudit.org/revue/rse/2005/v31/n2/012757ar.pdf

Schön, D. A. (1983). Le praticien réflexif. À la recherche du savoir caché dans l'agir professionnel (J. Heynemand \& D. Gagnon, trad.). Montréal : Logiques.

Sensevy, G. et al. (2013). Les lieux d'éducation associés à l'IFé (LéA) : quelles recherches à y développer? Bulletin de la recherche l'IFé, 17, 1-2.

Shulman, L. (1986). Those who understand: Knowledge growth in teaching. Educational Researcher, 15 (2), 4-14.

Vinatier, I. (2007). Un dispositif de «co-explicitation» avec chercheur et maîtres formateurs : la conceptualisation de l'activité de conseil. Actes du Congrès Actualité de la recherche en éducation et en formation (AREF), Strasbourg, Fr. Téléaccessible à : http://www.congresintaref.org/actes pdf/AREF2007_Isabelle VINATIER 229.pdf

Vinatier, I. (2009). Pour une didactique professionnelle de l'enseignement. Rennes: Presses Universitaires de Rennes.

Yvon, F. \& Durand, M. (2011). Réconcilier recherche et formation par l'analyse de l'activité. Bruxelles : De Boeck Supérieur, «Perspectives en éducation et formation». 